\title{
Development of core-collections for Guizhou tea genetic resources and GWAS of leaf size using SNP developed by genotyping-by-sequencing
}

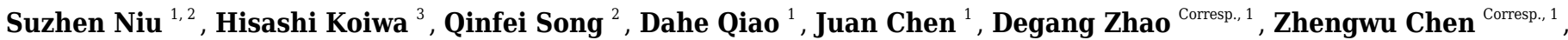 \\ Ying Wang ${ }^{4}$, Tianyuan Zhang ${ }^{4}$ \\ ${ }^{1}$ Guiyang Station for DUS Testing Center of New Plant Varteties (MOA) / Institute of Tea, Guizhou Academy of Agricultural Sciences, Guiyang, China \\ 2 The Key Laboratory of Plant Resources Conservation and Germplasm Innovationin Mountainous Region (Ministry of Education), Institute of Agro- \\ Bioengineering / College of Tea Science, Guizhou University, Guiyang, China \\ 3 Vegetable and Fruit Improvement Center, Department of Horticultural Sciences, Molecular and Environmental Plant Sciences Program, Texas A\&M \\ University, College Station, Texas, USA \\ 4 Wuhan Benagen Tech Solutions Company Limited, Wuhan, China \\ Corresponding Authors: Degang Zhao, Zhengwu Chen \\ Email address: dgzhao@gzu.edu.cn, zwchentea@163.com
}

An accurate depiction of the genetic relationship, the development of core collection, and genome-wide association analysis (GWAS) are key for the effective exploitation and utilization of genetic resources. Here, genotyping-by-sequencing (GBS) was used to characterize 415 tea accessions mostly collected from the Guizhou region in China. A total of 30,282 high-quality SNPs was used to estimate the genetic relationships, develop core collections, and perform GWAS. We suggest 198 and 148 accessions to represent the core set and mini-core set, which consist of $47 \%$ and $37 \%$ of the whole collection, respectively, and contain $93-95 \%$ of the total SNPs. Furthermore, the frequencies of all alleles and genotypes in the whole set were very well retained in the core set and mini-core set. The 415 accessions were clustered into 14 groups and the core and the mini-core collections contain accessions from each group, species, cultivation status and growth habit. By analyzing the significant SNP markers associated with multiple traits, nine SNPs were found to be significantly associated with four leaf size traits, namely MLL, MLW, MLA and MLSI $(P<1.655 \mathrm{E}-06)$. This study characterized the genetic distance and relationship of tea collections, suggested the core collections, and established an efficient GWAS analysis of GBS result. 


\section{Development of core-collections for Guizhou tea genetic resources and}

\section{GWAS of leaf size using SNP developed by genotyping-by-sequencing}

3 Suzhen Niu ${ }^{1,2,3}$, Hisashi Koiwa ${ }^{3}$, Qinfei Song ${ }^{2}$, Dahe Qiao ${ }^{1}$, Juan Chen ${ }^{1}$, Degang Zhao ${ }^{1,2}$, Zhengwu Chen ${ }^{1}$, $4 \quad$ Ying Wang ${ }^{4}$, Tianyuan Zhang ${ }^{4}$

$5{ }^{1}$ Guiyang Station for DUS Testing Center of New Plant Varteties (MOA) / Institute of Tea, Guizhou Academy of 6 Agricultural Sciences, Guiyang 550006, Guizhou Province, RP China

$7 \quad{ }^{2}$ The Key Laboratory of Plant Resources Conservation and Germplasm Innovationin Mountainous Region (Ministry

8 of Education), Institute of Agro-Bioengineering / College of Tea Science, Guizhou University, Guiyang 550025,

9 Guizhou Province, RP China

$10{ }^{3}$ Vegetable and Fruit Improvement Center, Department of Horticultural Sciences, Molecular and Environmental

11 Plant Sciences Program, MS2133 Texas A\&M University, College Station, 77843-2133, TX, USA

$12{ }^{4}$ Wuhan Benagen Tech Solutions Company Limited, Wuhan 430070, Hubei Province, RP China

13 Corresponding Author: Degang Zhao, Email:dgzhao@gzu.edu.cn; Zhengwu Chen, Email: zwchentea@163.com 


\section{Abstract}

15 An accurate depiction of the genetic relationship, the development of core collection, and genome-wide association analysis (GWAS) are key for the effective exploitation and utilization of genetic resources. Here, genotyping-by-sequencing (GBS) was used to characterize 415 tea accessions mostly collected from the Guizhou region in China. A total of 30,282 high-quality SNPs was used to estimate the genetic relationships, develop core collections, and perform GWAS. We suggest 198 and 148 accessions to represent the core set and mini-core set, which consist of $47 \%$ and $37 \%$ of the whole collection, respectively, and contain $93-95 \%$ of the total SNPs. Furthermore, the frequencies of all alleles and genotypes in the whole set were very well retained in the core set and mini-core set. The 415 accessions were clustered into 14 groups and the core and the mini-core collections contain accessions from each group, species, cultivation status and growth habit. By analyzing the significant SNP markers associated with multiple traits, nine SNPs were found to be significantly associated with four leaf size traits, namely MLL, MLW, MLA and MLSI $(P<1.655 \mathrm{E}-06)$. This study characterized the genetic distance and relationship of tea collections, suggested the core collections, and established an efficient GWAS analysis of GBS result. 


\section{Introduction}

Tea, coffee and cocoa are considered as the world's three best-known beverages and are also the most produced or consumed beverages worldwide (Wambulwa et al., 2016; Liu et al., 2017). To produce tea, people need to infuse processed tender shoots of the tea plant (Camellia sinensis) with connected species, all of which belong to Thea of genus Camellia in the family Theaceae (Ma et al., 2018). Tea is a diploid $(2 \mathrm{n}=2 \mathrm{x}=30)$ with a genome size of $3.02 \mathrm{~Gb}$ and is an endemic species in southwest China (Wei et al., 2018). Tea is highly nutritious with medicinal properties, offering a wealth of health benefits. Daily tea consumption is beneficial for reducing the risks of various cancers, diseases related to obesity, and neurological and cardiovascular dysfunctions (Naghma et al., 2013; Hayat et al., 2015). The rich flavors and multiple healthpromoting functions of tea are conferred by 700 bioactive compounds such as catechins, caffeine, theanine, and volatiles (Xia et al., 2017).

Tea has a long utilization history in the Yunnan-Guizhou Plateau and is now cultivated worldwide (Wei et al., 2018; Chen et al., 2012). In this region, the diversity of tea germplasm is well preserved with abundant wild tea plants, ancient landraces and modern landraces with different morphological characteristics due to the region's unique geology, diverse climates, plentiful rainfall and the cross-pollination nature of tea (Niu, 2014). In addition, due to the slow socio-economic development and land use in Guizhou Plateau, elimination of various tea species at a large spatial scale has not occurred.

The ever-growing popularity of tea products calls for new tea varieties that meet the needs of market diversification (Liang et al., 2015). Similar to other perennial woody species, tea plants have a long growth period and the genome is highly heterozygous, making breeding difficult and costly. Tea producers also face new challenges such as the sustainability of high-quality tea production, environmental change, pest invasion, and diseases. Hence, it is vital to explore the genetic basis of complex traits and identify favorable alleles for breeding new tea cultivars that can overcome these threats by marker-assisted breeding (MAB) (Tan et al., 2016).

Compared with other ways, association mapping is faster and more effective in dissecting 
58 the genetic basis of complex traits and identifying favorable genetic resource. The results yielded

59

60 from association mapping have greatly facilitated MAB programs (Iso-Touru et al., 2016). Association mapping is also promising in evading limitations of linkage mapping. The difference between traditional linkage mapping which is often based on bi-parental populations and association mapping is that the latter takes advantage of the ancestral recombination in natural populations to identify loci that significantly associate with traits of interest based on the linkage disequilibrium (LD) (Buckler et al., 2002; Pace et al., 2015). Analysis of a large number of alleles in various populations is made possible by association mapping (Pace et al., 2015; Suwarno et al., 2015; Motilal et al., 2016). Other advantages of GWAS compared with traditional linkage mapping (Tan et al., 2016; Bali et al., 2015) include high-resolution and providing a less time-consuming approach for developing the mapping population (Heena et al., 2018).

The population used for association mapping is of great importance, it must have a wide range of diversity that represents most historical recombination events (Heena et al., 2018). Although species diversity with abundant tea genetic collections is well preserved in the center of origin, recent tea breeding programs have not exploited the allelic diversity of many traits. As a result, characterizing tea plant collections should be the first step towards stimulating the use of genetic resources. Germplasm preservation can be costly and time-consuming, therefore, developing 'core collections' with a minimal number of tea varieties that retain the genetic diversity is a cost-effective strategy (Frankel.,1984; Brown.,1989; Wang et al.,2011; Taniquchi et al., 2014; Campoy et al.,2016; Hintum et al.,2000; Ndjiondjop et al., 2017; Lassois et al.,2016). In addition, 'core-collections' are useful for genetic association analysis and the identification of genomic variation (Hugginsa et al., 2018; Zhang et al., 2018; Muleta et al., 2016; Cunff et al., 2008; Heena et al., 2018; Ma et al., 2018). Criteria based on the genetic distance between accessions have been proven sueful in evaluating and creating 'core collections' (Odong et al., 2013; Campoy et al., 2016; Ndjiondjop et al., 2017).

Previous studies on association mapping and core-collection development mainly focused on 
85

86

maize (Pace et al., 2015; Suwarno et al., 2015; Coan et al., 2018), sunflower (Heena et al., 2018), wheat (Muleta et al., 2017), pine (Bartholome et al., 2017), sorghum (Bouchet et al., 2017), finger millet (Babu et al., 2018), melon (Hou et al., 2018), pea (Desgroux et al., 2018) and cotton (Ma et al., 2018). By contrast, little has been done on tea germplasm with high density SNPs distributed on the whole genome. GBS is a large-scale approach for identifying high density SNP markers that are suitable for association mapping. In this study, GBS was used to analyze the genetic diversity and population structure of 415 tea accessions including wild types, ancient and modern landraces from the Guizhou Plateau, and breeding varieties from Zhejiang, Fujian, Hunan and Guizhou. We aim to (1) analyze the genetic purity, distance and relationships, (2) establish and evaluate the core collections, and (3) perform GWAS on the tea population. Our findings provide a valuable resource for developing new molecular markers that can be used for MAB of tea varieties.

\section{Materials and Methods}

\section{Plant materials}

A total of 415 accessions including 159 wild type and 256 cultivation type varieties (174 ancient landraces, 77 modern landraces and 5 breeding varieties) were used for this study (Fig. S1; Table. S1). Based on the classification system established by Chen et al. (2000) and Min (1992), 251 C.sinensis (L.) O. Ktze, 100 C.tachangensis F.C. Zhang, 59 C. remotiserrata Zhang, and five near C.taliensis W.W. Smith were identified (Table S1). Hereafter, wild varieties and their natural offspring will be referred to as "wild type", the cultivation tea varieties aged more than one hundred years old will be called "ancient landraces", and the tea garden landraces will be named "modern landraces" (Table. S1). The ancient landraces, modern landraces and "breeding varieties" that have undergone artificial selection are collectively referred to as the "cultivation type".

One hundred and sixty-eight samples were collected from areas suitable for tea growth in north Guizhou (Ia) (Fig. S1), 51 samples were collected from areas suitable for tea growth in east 
112 Guizhou (Ib), 57 samples were collected from areas suitable for tea growth in south Guizhou (Ic),

11383 samples were collected from areas suitable for tea growth in central Guizhou (II), 41 samples

114 were collected from areas with a minor suitable climate for tea growth in west Guizhou (III), ten

115 samples were collected from areas with an unsuitable climate for tea growth in west Guizhou,

116 one cultivar was collected from Guizhou, two cultivars were collected from Fujian, one cultivar

117 was collected from Zhejiang, and one cultivar was collected from Hu'nan (Table. S1). The

118 samples were planted in the city of Guiyang, China. The healthy tender shoots were harvested,

119 snap-frozen in liquid $\mathrm{N}_{2}$, and kept at $-80{ }^{\circ} \mathrm{C}$ until use (Jin et al., 2018).

120

121

122

123

124

125

126

127

128

129

130

131

132

133

134

135

136

137

138

\section{DNA extraction}

Genomic DNA was extracted from the plant samples with a kit for rapid extraction of genomic DNA. DNA integrity was tested by 1\% agarose gel electrophoresis; a Qubit Fluorometer was used to check the purity and measure the concentration of DNA samples (Niu et al., 2019).

\section{Sequencing of the GBS library}

One-hundred nanogram genomic DNA was double digested with $5 \mathrm{U}$ of SacI and MseI (NEB) in a $25 \mu 1$ reaction containing $1 \times$ restriction buffer. The resulting samples were ligated with the restriction fragment using the SacAD and MseAD adaptors, which contained different barcode combinations to distinguish the samples. Equal volumes of the ligated products of 12 individuals were pooled and processed using the QIA quick PCR Purification Kit (Qiagen). PCR was performed using PCR Master Mix and the PCR Primer Cocktail, which could enrich DNA fragments with the adapters. The PCR products of each mixture were pooled and separated on a $2 \%$ agarose gel by electrophoresis. Fragments of 500 to $550 \mathrm{bp}$ (including the $120 \mathrm{bp}$ adaptor) were recovered with the QIA quick Gel Extraction Kit (Qiagen). The average length of DNA fragment was selected for final library construction with the Agilent DNA 12,000 kit using the 2100 Bioanalyzer system. To quantify the final library, quantitative real-time PCR with a Taq Man probe was employed. Then the libraries were sequenced on the IlluminaHiSeq X Ten platform based on the paired-end 150 (PE150) strategy. Each library contains 48 samples, and clean data were then parsed into different units that exactly match the barcodes and the 
139

140

141

142

143

144

145

146

147

148

149

150

151

152

153

154

155

156

157

158

159

160

161

162

163

164

restriction sites at both ends (Elshire et al., 2011).

\section{SNP genotyping basing on the sequence data}

Original IlluminaHiSeq X Ten reads were de-multiplexed based on the barcodes, and a custom

Perl script was used to shear the barcoded sequences. Reads in which $>50 \%$ of the bases have quality values $\leqslant 5$ were discarded; the clean reads were mapped to the tea reference genome (http://www.plantkingdomgdb.com/tea tree/) (Xia et al., 2017) using BWA-MEM (v.0.7.10) with parameters '-T $20-k$ 30' (Li, 2013). SNPs and InDels were called using GATK (v.3.7.0).

The SNPs were filtered according to the methods described by Hussain et al (2017), Chen et al (2017) and Eltaher et al. (2018) based on the following criteria: (1) variants must be bi-allelic SNPs, (2) "QUAL < 50.0 || QD < 2.0 || FS > 60.0 || MQ < 40.0 || Mapping Quality Rank Sum < 12.5 || Read Pos Rank Sum < -8.0" was used in Variant Filtration in GATK (V 3.7.0) to filter the SNPs, (3) SNPs with MAF $<0.05$ or $>10 \%$ missing data were filtered out by VCFtools (V 0.1.15); (4) a window of 50 SNPs, a step size of ten SNPs, and an $\mathrm{r}^{2}$ threshold of 0.2 were used to prune the SNPs using Plink (v1.9). As a result, a set of 415 accessions and 30,282 high-quality SNPs were retained and used for subsequent analyses (Table S2). We connected all the scaffolds into 20 pseudo-groups (Table. S7).

\section{Phenotypic data collection}

The 415 accessions were measured for leaf size for association mapping. We measured the ten representative mature leaves length (MLL) and mature leaves width (MLW) of each individual and calculated their means to represent the traits of MLL and MLW, respectively. Leaf size measurements were performed in Spring (monthly average temperature is $14.68^{\circ} \mathrm{C}$ ) and Fall (monthly average temperature is $21.29^{\circ} \mathrm{C}$ ), 2018, seperately. Leaf shape index (MLSI), calculated as MLL/MLW, was the third trait we measured. The fourth trait, mature leaf area $($ MLA), was calculated as $0.75 \times$ MLW $\times$ MLL. We modified our data collection method according to guidelines of UPOV (International Union for the Protection of New Varieties of Plants 2008) for tea. Statistical analysis of the phenotypic data was conducted using Microsoft 
165

166

167

168

169

170

171

172

173

174

175

176

177

178

179

180

181

182

183

184

185

186

187

188

189

190

Excel 2010 and SPSS 15.0 software. Pearson's correlation coefficients (r) were calculated for the four traits. Means, standard deviations, and ranges were calculated for trait distribution. To evaluate whether the data followed a normal distribution, Skewness and Kurtosis were calculated using the Descriptive Statistics model-based Frequencies distribution of analysis implemented in SPSS15.0 software.

\section{Genetic purity, distance, and relationships of accessions}

The following equation was used to calculate the polymorphism information content (PIC) values for the SNP data (Bostein et al., 1980). $P i$ and $P j$ were the frequencies of the number of $i$ and $j$ alleles respectively, and $\mathrm{n}$ was the number of alleles (Botstein et al.,2008). We can evaluate each marker locus for its PIC by summing the mating frequencies multiplied by the probability that an offspring will be informative. Under our assumptions, the expected value of PIC can be calculated as

$$
\mathrm{PIC}=1-\sum_{i=1}^{n} P_{i}^{2}-\sum_{i=1}^{n-1} \sum_{j}^{n} 2 \mathrm{P}_{\mathrm{i}}^{2} \mathrm{P}_{\mathrm{j}}^{2}
$$

The alleles, genetic distance and the observed heterozygosity (Ho) were calculated on every groups with TASSEL v.5.2.37 (Bradbury et al., 2007). The neighbor-joining cluster analysis were performed using DARwin v.6.0.17.

\section{Development of Core Collections}

As subsets of larger genetic collections, core collections contain the smallest number of accessions that represent the maximum diversity of the raw collection. DARwin (v.6.0. 17) was used to construct the diversity trees (Hamon et al., 2003). We used 10,000 bootstraps to determine dissimilarities and transformed them into Euclidean distances. The phylogenetic tree was constructed based on the 30,282 SNPs using the unweighted Neighbor Joining (NJ) method. Then, the 'maximum length subtree function' was used to generate the core collection as described previously for cowpea (Egbadzor et al., 2014), prunus (Campoy et al., 2016), sorghum (Claire et al., 2013) and rice (Ndjiondjop et al., 2017). The maximum length subtree implemented a stepwise procedure that consecutively prunes redundant units. In this procedure, 
191

192

193

194

195

196

197

198

199

200

201

202

203

204

205

206

207

208

209

210

211

212

213

214

215

216

217

sample size determination, which maintains the largest diversity, is allowed, and the procedure is visualized in the phylogenetic tree of the original population of all 415 accessions. If the distance between two accessions, as judged by the edge length, is small, they are considered redundant. Because of more uncommon characters, accessions with the longest edge are considered the most diverse. A 'removed edge value' offered by the NJ tree was used to identify presumptive clusters of synonym accessions, and a threshold value of 0.0008 (Euclidean distance) was required to confirm the synonym mentioned. We determined the final core set that represents the maximum genetic diversity based on the pruned edge length of the initial tree length and the sphericity index (Hamon et al., 2003).

\section{Association analysis}

Association analysis was carried out on the four measurable traits, namely MLL, MLW, MLSI, and MLA, in two seasons, independently. We used the mixed linear model (MLM) implemented in TASSEL (v.5.2.37) because the GLM model has a high false positive rate (Bradbury et al., 2007) following the user manual. To overcome this limitation, a comparison was drawn between the two association analysis models using TASSEL 5.2.43, and the most optimized model for each analyzed quality trait was identified and used for subsequence analyses (Bradbury et al., 2007; Heena et al.,2018). To control the potential false-positives result from the confounding of population structure, PCA-matrix or Q-matrix was used as the fixed effect in MLMs. Kinship matrix $(\mathrm{K})$ was considered as a covariate factor in MLMs to cut down the rate of false positives among genotypes (Yu et al., 2006). If the significance threshold of a SNP has the lowest $P$-value in the peak area $\left(P \leqslant 0.05 / 30,282=1.65 \times 10^{-6}\right)$, it is considered significantly associated with the targeted trait. Correlation coefficient $\left(\mathrm{R}^{2}\right)$ explained the phenotypic variation by every markertrait association analyzed.

\section{Results}

Genetic purity, distance, and relationships

GBS analysis of the 415 tea accessions was conducted using IlluminaHiSeq $X$ Ten and the relevant information was described in our previous study (Niu et al., 2019). Observed 
218 heterozygosity in each sample ranged from 0.041 to 0.386 (Fig. S2), with an average 219 heterozygosity of 0.225 . Among the 415 accessions, $73.5 \%$ had an observed heterozygosity $>$ 2200.201 (Table. S1). The pairwise genetic distance between two accessions ranged from 0.088 to 2210.298 (Table. S2), with an average genetic distance of 0.249 among all accessions (Fig. 1; Table. 222 S2). The genetic distances of $36.6 \%$ accession pairs were between 0.200 and 0.250 , and those of $2233.73 \%$ and 59.68\% accession pairs were $<0.200$ and $>0.250$, respectively (Fig.1; Table S2). 224 Based on the NJ cluster analysis on the genetic distance matrix, the 415 accessions could be clustered into fourteen groups (Fig. 2A; Table. S1) based on cultivation status (ancient landraces, wild tree, modern landraces and breeding cultivars), growth habits (cultivation type and wild type), and species, and the here-derived core and mini-core collections of origin. Group one consisted of 71 accessions, most of which were cultivation type (of the group one, $85.92 \%$ are cultivation type), ancient landraces (of the group one, $74.65 \%$ are ancient landraces), and $C$. sinensis (of the group one, $85.92 \%$ are $C$. sinensis). The second group contained 88 accessions, most of which belong to $C$. tachangensis (of second group, 93.18\% are C. tachangensis) and wild type (of the second group, 96.59\% are wild type). The third group consisted of 54 accessions, most of which were wild type (of the third group, 98.15\% are wild type) and $C$. remotiserrata varieties (of the third group, 91.48\% are C. remotiserrata). Group four had a total of 76 accessions and most were cultivation type (of the group four, $97.37 \%$ are cultivation type), modern landraces (of the group four, $61.84 \%$ are cultivation type) and $C$. sinensis (of the group four, 98.68\% are C. sinensis). Group five had 54 accessions, all of which were from $C$. sinensis and cultivation type, and the number of modern landraces were almost the same as that of the ancient landraces. Groups six, seven, eight, 12, 13 and 14 consisted of 15, 15, two, seven, one and one accessions, respectively, all of which were ancient landraces, cultivation type, and $C$. sinensis varieties. Groups nine and ten consisted of three and six accessions, respectively, which were wild tree and C. remotiserrata varieties. Group 11 had a total of 20 accessions and most were cultivation type (of the Group 11, 95.45\% are cultivation type), ancient landraces (of the Group 11, 95.45\% are ancient landraces) and $C$. sinensis varieties (of the group $11,90.91 \%$ are $C$. 
245

246

247

248

249

250

251

252

253

254

255

256

257

258

259

260

261

262

263

264

265

266

267

268

269

270

271

sinensis varieties) (Fig. 2A; Table. S1).

\section{Creating core and mini-core collections}

We proposed genetic core and mini-core sets to represent the genetic diversity of a large tea population. These core and mini-core sets can be used for association studies, breeding, and other purposes (Brown, 1989). The maximum length subtree method implicated in DARwin v.6.0.17. was repeatedly applied to remove the most redundant accessions until the sphericity index percentage and pruned edge were linear with a relatively low slope, corresponding to 299 accessions (Fig. S4). The 299 core accessions could represent the 415 accessions (referred to as 'core-1' from hereafter) (Table S1; Fig. 2B). Then, the sphericity index increases stably and slowly from 301 to 198 on the x-axis, indicating no significant difference in the information of the 104 accessions, suggesting that the elimination of these accessions had no significant impact on sphericity index (Fig. S4). The 198 core accessions (referred to as 'core-2' hereafter, which contains less accessions than 'core-1') were selected to represent the 415 accessions (Fig. 2C; Table. S1). The percentage of sphericity index and pruned edge flattened until reaching a sample size of 146 accessions on the $\mathrm{x}$-axis (Fig. S4). Thus, the 146 remaining accessions constitute the mini-core set. We suggest that these 148 accessions, consisting of the 146 retained accessions and two cultivars ('Fudingdabaicha' and 'Tieguanyincha'), represent the mini-core set of the Guizhou Plateau tea germplasm (Fig. 2D; Table. S1). The selected cores and mini-core sets originated from 14 different clusters yielded from NJ tree, respectively, although some individuals were assigned to other clusters (Fig. 2), which suggested that edges constituting the phylogenetic tree backbone have been retained.

\section{Diversity assessment of the cores and mini-core sets}

The alleles and genotypes, PIC, genetic distances and minor allele frequency of the 415 accessions were compared to those of the two core sets and the mini-core set. With regard to the average polymorphic sites in the 415 tea accessions, the reduction in sample size from 415 to 299 and 198 accessions in the two core sets, and 148 in the mini-core set reduced SNPs numbers by only $103(0.36 \%), 1,403(4.93 \%)$, and 1,722 (6.04\%), respectively (Table. 1). Ho, PIC and 
272 MAF in Core-1 and Core-2 sets were almost the same as for whole tea accessions, suggesting 273 that both core- 1 and core- 2 can represent the 415 accessions. Ho, PIC and MAF in mini-Core set 274 were $89.77 \%, 98.89 \%$ and $98.25 \%$ of those of the whole population, respectively (Table. 1). 275 Allele and genotype frequencies in the two core sets and one mini-core sets almost made no odds 276 from the entire set (Fig. S3). Consistent with our hypothesis, the genetic distances between 277 accession pairs increased slightly in the core and mini-core sets (Fig. 1; Table S2 S5). The 278 proportion of accession pairs with a genetic distance greater than 0.15 was $98.59 \%$ for the entire 279 population, compared to $99.95 \%, 99.97 \%$ and $99.95 \%$ in core-1, core-2 and the mini-core set 280 (Fig. 1; Table. 1), respectively. The proportion of samples number removed per group based on cluster analysis of NJ tree was not consistent in the two core and one mini-core sets (Fig. 2;

282 Table. 2; Table. S1). The proportion of accessions removed from group two and group four were 283 significantly higher than that from groups $1,3,5,6,7,10,11$ and 12 . The numbers of accessions

284

285

286

287

288

289

290

291

292

293

294

295

296

297

298

excluded from groups 8,9,13 and 14 in the two core sets and the mini-core set were same as that from the entire tea collection (Fig. 3). The mini-core collection consisted of the accessions from all 14 groups, as revealed by the cluster analysis of NJ tree, and represented 14 groups, three cultivation status, two growth habits, and four different species (Fig. 3). These results indicated that the core and mini-core collections can well represent the 415 accessions.

\section{Genome-wide association studies}

Skewness and Kurtosis for MLL, MLW and MLSI did not differed from those of a normal distribution (Table. 3). Despite the existence of left obliqueness, MLA still exhibited normal distribution. (Table. 3). The four traits were significant correlated $(P<0.0001)$, as revealed by the Pearson correlation analysis (Table. 4). MLSI was highly correlated with MLL and MLW but not with MLA. The same trait was significant correlated $(P<0.0001)$ between two environments (Table. 4).

The population structure of the 415 accessions was studied with STRUCTURE 2.3.4 on the basis of the LD-pruned 1,135 high-quality SNPs. Three subpopulations were acquired, which was coincident with the preceding reports (Niu et al., 2019). As a result, the Q-matrix was 
299

300

301

302

303

304

305

306

307

308

309

310

311

312

313

314

315

316

317

318

319

320

321

322

323

324

325

generated from $\mathrm{k}=3$ using STRUCTURE 2.3.4 and used for GWAS as the fixed effect. P-matrix, as the first three PCs value given by the PCA analysis, was used as covariates in the GWAS model. We also calculated kinship matrix using TASSEL. Therefore, the MLMQ+K and MLMP+K models were compared based on the Q-Q plot (Fig. S5) and the MLMP+K model best fitted the four traits.

We identified nine SNPs that significantly associated with MLL, MLW, MLA and MLSI ( $P$ $<$ 1.655E-06) (Fig. S6; Table. 5; Table. S6). Among these, three were significantly associated with multiple traits. In the case of MLL, significant associations were detected for SNPs PG_5:79474508 (on xfSc0000122) and PG_1:32346865 (on Sc0000000), which explained 3.77\% and $3.23 \%$ of the phenotypic variation, respectively, and had a deduced effect of major alleles from -0.72 to -2.94 (Table. 5; Table. S6).

For MLW, only one significant SNP was detected on the same locus as MLL, which explained $8.24 \%$ of the phenotypic variation; this SNP has a negative major allele effect of -1.31 and a positive minor allele effect of 0.44 for MLW. Six loci, located at Sc0000000, Sc0000365, Sc0002172, Sc0002452, Sc0004093 and xfSc0000122, significantly correlated with MLA, explaining $3.49 \%$ to $4.50 \%$ of the total phenotypic variation, with the main influence varying from $-18.23 \%$ to $4.462 \%$ and minor effects varying from $-18.76 \%$ to $42.47 \%$ (Table. 5; Table. S6). Three SNPs located at Sc0000037, Sc0000011 and Sc0000046 were found to significantly associate with MLSI, explaining 4.012\%, 4.33\%, and 3.83\% of the MLSI variation at 82,813,415, $30,871,283$, and $98,891,957 \mathrm{bp}$, respectively; while the deduced effect of the major alleles ranged from -0.08 to 0.06 (Table. 5; Table. S6). Among these SNPs, two were co-associated with multiple traits. PG_5:79474508, which had a negative effect of major alleles, was co-associated with MLL and MLA. PG_1:32346865 with an increased effect of major alleles was coassociated with MLL, MLW and MLA (Table. 5; Table. S6). Three, three and two significantly associated SNPs identified in the whole population were retained in core-1, core-2 and mini-core set, separately (Table. S7).

\section{Discussions}


326 Genetic purity, genetic distance and relationships

327 GBS is a cost-efficient and effective genotyping method (Yang et al., 2017; Bhattarai et al., 2018;

328 Hackett et al., 2018). In this study, $390.30 \mathrm{~Gb}$ clean reads were generated. Among them, 0.208-

$3293.32 \mathrm{~Gb}$ clean reads were generated after the quality filtering step in 415 accessions, and 30,282

330 high-quality SNPs were retained with strict filter conditions. More SNPs were identified in this 331 study compared with previous studies, which meets the requirements of our objectives (Chen et 332 al., 2017; Eltaher et al., 2018)

333 Camellia sinensis and related species are cross-pollinated species with a low self-pollination 334 rate ranging from $2 \%$ to $6 \%$ (Chen et al., 2000; Ma et al., 2018). Consistently, the heterozygosity 335 rate of $73.5 \%$ were $>20 \%$. Most alterations in allele frequencies occurred during natural 336 regeneration without human intervention, which is in agreement with previous studies (Niu, 337 2014), indicating that the tea population from the center of origin maintained a high level of 338 genetic diversity. Genetic distance measures the genetic divergence between a given pair of 339 accessions or populations; the pairs usually share many alleles with a small genetic distance 340 (Ndjiondjop et al., 2017). The results in this study suggested remarkably different genetic 341 distances between pairs of 415 accessions, with $3.73 \%$ of the pairs in similarity, $36.6 \%$ of the 342 pairs moderately distant, and 59.68\% significantly distant. We detected very low redundancy in 343 our collection, which consists with the low observed genetic divergence and high genetic 344 variation retained from the original tea population. Using clustering, we identified 14 groups or clusters to establish the core accessions. In general, the groups clustered according to the cultivation status, growth habits, and species.

347 Selection of core and mini-core

348 Establishing the core and the mini-core sets with the lowest level of redundancy that represent 349 the maximum potential genetic diversity from the total collection facilitates the identification of 350 suitable variations for GWAS and MAB (Brown, 1989; Ndjiondjop et al., 2017). Core 351 collections could be evaluated based on genetic markers or phenotypic traits, including pairwise 352 distances (Hamon et al., 2003; Franco et al., 2005; Leroy et al., 2014) and allelic 
353

354

355

356

357

358

359

360

361

362

363

364

365

366

367

368

369

370

371

372

373

374

375

376

377

378

379

richness/diversity (Beukelaer et al., 2012; Beukelaer et al 2018). Selection of the most suitable evaluation method depends upon the purpose of core collections (Odong et al., 2013). Preserving most alleles is an ideal way to conserve germplasm, while the approaches based on distance majorly hammer the retention of most combinations of alleles in specific genotypes, which are suitable for GWAS and MAB (Campoy et al., 2016; Ndjiondjop et al., 2017). Thus, we used the distance-based methods applied on GBS data to propose the first core collection and the minicore collection from the tea origin center, the Guizhou plateau, and accounting for wild trees, ancient landraces, modern landraces and cultivars in this study. Core-1 and Core- 2 collections and one mini-core collection were created based on Sphericity index and the length of pruned values, which contain $72 \%, 48 \%$ and $36 \%$ of the total number of accessions. Ho, PIC, MAF, allele and genotype frequencies in Core- 1 and Core- 2 sets were almost the same as for the whole population, which suggested that both core- 1 and core- 2 can well represent the 415 accessions for further study. The genetic diversity parameters, genetic distances, allele information and retained SNPs of core-1 and core- 2 revealed that the core- 2 of 198 accessions was equally appropriate for representing the whole population as core-1 of 299 accessions Therefore, the core-2 of 198 accessions was chosen as the appropriate set, considering the costs of future research. The mini-core set selected in this study is easy to manage for phenotypic and physiological evaluation in the field and under controlled conditions when selecting parent lines for improving the traits of interest and identifying genes associated with these traits using GWAS.

\section{Genome-wide association studies}

Based on morphology, tea plants can be divided into two subgroups. Small-leaf shrubs that are cold tolerant constitute group one, whereas group two consists of large-leaf arbor trees that are less resistant to cold (Yao et al., 2011). Leaf size is associated to the fitness and temperatureadaptation history of tea varieties (Tan et al., 2016). Leaf size may also relate to tea production and the shape of dry tea. The mapping population used in this study included two sub groups based on leaf size. Therefore, we observed a high level of segregation in all four leaves sizerelated traits. Quantitative leaf traits are influenced by the environment (Tan et al., 2016; Baker 
380

381

382

383

384

385

386

387

388

389

390

391

392

393

394

395

396

397

398

399

400

401

402

403

404

405

406

et al., 2016). In this study, the same trait was significantly correlated between two different environments, suggesting that there are little false positive in the significantly associated SNPs.

MLM was employed for association mapping and two models (MLMQ+K and MLMP+K) to control false positive caused by population structure. Leaf size is associated with local adaptation, and it can actually decrease the false rate by removing accessions with extreme genetic diversity and phenotypic expression (Pace et al., 2015). We did not test the GLM model but used the combination of MLMQ+K and MLMP+K models, which reduce the rate of false positives for detecting SNPs, and are proven to be more successful than using each strategy alone (Song et al., 2019). Q denotes the Q-matrix produced from the population structure $(\mathrm{k}=3)$ and $\mathrm{P}$ represents P-matrix from the top three PCs, that are used as covariates in GWAS. K refers to kinship matrix and is used to determine the correlations between individuals. Both the $\mathrm{Q}+\mathrm{K}$ and $\mathrm{P}+\mathrm{K}$ matrices fit the MLM to control spurious associations resulted from relatedness and population structure, separately (Zhao et al., 2019).

In previous studies, tea germplasm showed significant variations for leaf size (Yao et al., 2011). However, to our knowledge, few genetic loci influencing this trait have been identified. Tan et al. (2016) reported one major QTL (qLSI13) for leaf size in tea plant. In this study, nine SNPs significantly associated with leaf traits were identified using the P+K MLM. Six, three, two and one SNPs were significantly associated with MAL, MLSI, MLL and MLW, respectively. SNP PG_1:32346865 was significantly associated with three traits, MLL, MLW and MLA, all were significantly positively correlated $(P<0.0001)$, Which was consistent with the results of significant positive correlation among the three characters (Table. 4). Three SNPs significantly associated with MLSI were carrying with only 1 minor allele, which need to keep the work in exploring the truth in next step study.

Our study provides evidence that leaf size is affected by major effect genes, which was consistent with previous reports (Tan et al., 2016). These results lay a foundation for future study and will inform leaf size-related gene mining and MAB in tea breeding.

\section{Conclusions}

Peer) reviewing PDF | (2019:05:38114:2:0:NEW 25 Dec 2019) 
407 This study illustrates that GBS is efficient for analyzing the genetic purity, distance and 408 relationship of tea germplasm, and for creating the core sets. The core and mini-core collections 409 account for approximately $47 \%$ and $37 \%$ of the entire collection, respectively, and contains $93-$ $41095 \%$ of all SNPs and almost all genotype frequencies and alleles that were observed in the entire 411 tea collection. These core sets are highly valuable for identifying favorable alleles and selecting 412 parent lines to improve agronomically important traits in tea varieties. Our study identified SNP 413 markers associated with leaf size. These SNPs can be employed in MAB for tea improvement.

\section{Figure and Table legends}

415 Fig. 1 Frequency distribution categories of pairwise genetic distance of 415 tea accessions (red 416 fill); a core set of 299 accessions (orange fill); a core set of 198 accessions (green fill), and a 417 mini-core set of 148 accessions (purple fill), based on 30,282 polymorphic SNPs.

418 Fig. 2 Neighbor joining tree for (A) 415 accessions (G1 = Group 1, pink; G2 = Group 2, red;G3= 419 Group 3, light green; G4 = Group 4, blue; G5 = Group 5, brown ; G6 = Group 6, orange red; G7 $420=$ Group 7 , orange yellow; G8 = Group 8, light blue; G9 = Group 9 ,yellow; G10 = Group 10, 421 light purple; G11= Group 11, dark green; G12 = Group12, dark blue; G13 = Group 13, black; 422 G14 = Group 14, purple), (B) 299 accessions selected for a core set, (C) 198 accessions selected 423 for a core set and (D) 148 accessions selected for a mini-core set based on 30,282 polymorphic 424 SNPs.

425 Fig. 3 Summary of accessions elected to conform core-1, core-2 and mini-core collection 426 compared with the whole tea accessions by (A) groups predicted based on cluster analysis (G1 = 427 Group 1,G2 = Group 2, G3= Group 3, G4 = Group 4, G5 = Group 5, G6 = Group 6, G7 = Group $4287, \mathrm{G} 8=$ Group 8, G9 = Group 9 , G10 = Group 10, G11= Group 11, G12= Group 12, G13= 429 Group 13, G14 = Group 14) ; (B) summary of accessions selected to account for core-1, core-2 430 and mini-core collection compared with the whole tea accessions in each group by cultivation 431 status (AL, Ancient landraces; ML, Modern landraces; WT, Wild tree ), by Growth Habits (CT, 432 cultivation type; WT, wild type) and Species of origin (S1, C.sinensis; S2, C.tachangensis; S3, 433 C.remotiserrata; S4, C.taliensis).

434 Table 1. Genetic differentiation of inferred populations of tea accessions from Guizhou 435 Province.

436 Table 2. The number of whole accessions and core collections distributed in 14 groups. 
437 Table 3. Trait statistics collected for mature leaf length (MLL), mature leaf width (MLW),

438 mature leaf shape index (MLSI) and mature leaf area (MLA).

439 Table 4. The correlation coefficient between two environment and among four traits.

440 Table 5. SNPs significantly associated with mature leaf length (MLL), mature leaf width

441 (MLW), mature leaf shape index (MLSI) and mature leaf area (MLA) detected by GWAS of 415 442 accessions

\section{Supplemental Figure and Table legends}

444 Fig. S1 Geographic distribution of tea accessions analyzed in this study according to the 445 collection.

446 Fig. S2 Frequency distribution of observed heterozygosity for 415 tea accessions based on 44730,282 polymorphic SNPs.

448 Fig. S3 Summary of (A) allele frequencies and (B) genotype frequencies of the entire tea 449 accessions $(\mathrm{N}=415)$ based on 30,282 polymorphic SNPs as compared with a core set of 299 450 accessions, a core set of 198 accessions, and a mini-core set of 148 accessions. Nucleotide codes 451 are as follows: A, adenine; C, cytosine; G, guanine; T, thymine; Y, C or T; R, A or G; K, G or T; 452 M, A or C; W, A or T; S, G or C (http://www.bioinformatics.org/sms/iupac.html; accessed in 453 Sept. 2017).

454 Fig. S4 Sphericity index and the length of pruned values for the selected core collection 455 individuals and mini-core collection for 415 tea accessions using 30,282 polymorphic SNPs.

456 Fig. S5 Q-Q plot of P-values analyzed using MLMQ+K and MLMP+K models. MLL: mature 457 leaf Length, MLW: mature leaf width, MLA: mature leaf area, MLSI: mature leaf shape index.

458 Fig. S6 Manhattan and quantile-quantile plots generated from GWAS results for leaf traits.

459 Table. S1 Information of 415 tea accessions used in this study, including the accession /clone 460 /collection, the accession name, the zone, the cultivation status, growth habits, the species, the 461 observed heterozygosity, the core collection $(\mathrm{N}=299)$, the core collection $(\mathrm{N}=198)$ and the mini462 core collection $(\mathrm{N}=148)$.

463 Table. S2 Identity-by-state (IBS)-based genetic distance matrix for 415 accessions using 30,282 464 polymorphic SNPs. 
465 Table. S3 Identity-by-state (IBS)-based genetic distance matrix for 299 accessions using 30,282 466 polymorphic SNPs.

467 Table. S4 Identity-by-state (IBS)-based genetic distance matrix for 198 accessions using 30,282 468 polymorphic SNPs.

469 Table. S5 Identity-by-state (IBS)-based genetic distance matrix for 148 accessions using 30,282 470 polymorphic SNPs.

471 Table. S6 The information of scaffold and SNP position in the genome Corresponding to the 472 name of pseudo-group (PG).

473 Table. S7 SNPs significantly associated with mature leaf length (MLL), mature leaf width 474 (MLW), mature leaf shape index (MLSI) and mature leaf area (MLA) detected by genome-wide 475 association study (GWAS) of core-1, core-2 and mini-core.

\section{Availability of data and material}

477 The 415 tea plant materials used in this study are planted in our germplasm resource nursery, and 478 the requests for materials should be addressed to Suzhen Niu (email: niusuzhen@163.com). 479 The raw sequence data reported in this study have been deposited in the Genome Sequence 480 Archive (Wang et al., 2017) in BIG Data Center, Beijing Institute of Genomics (BIG), Chinese 481 Academy of Sciences, under accession number CRA001438 that is publicly accessible at 482 http://bigd.big.ac.cn/gsa. Genotyping of 30,282 SNPs based on GBS in 415 tea accessions in this 483 study were deposited into the figshare website: https://doi.org/10.6084/m9.figshare.8019737.

\section{Competing interests}

485 The authors declare that they have no competing interests and consent for publication.

\section{Funding}

487 This work was supported by the National Science Foundation of China (31560222), the Science 488 and Technology Plan Project of Guizhou province (20172558, 20172557, 20175788, 20191404), 489 the USDA-NIFA SCRI grant (2017-51181-26834), the Guizhou Top Level Innovation Talents 490 Cultivation Project (20164003), the Talent Base for Germplasm Resources Utilization and 491 Innovation of Characteristic Plant in Guizhou (RCJD2018-14) and the Genetically Breeding 492 Major Project of the Ministry of Agriculture of China (2016ZX08010-003). 
493

494

495

496

497

498

499

500

501

502

503

504

505

506

507

508

509

510

511

512

513

514

515

516

517

518

519

\section{Authors' contributions}

S.Z.N, D.G.Z and Z.W.C conceived and supervised the study. H.K led writing and reviewed the manuscript. Q.F.S analyzed and interpreted GWAS. D.H.Q and J.C performed the DNA extraction and filtered the genotyping data. Y.W and T.Y.Z analyzed and interpreted Core Collection. All authors read and approved the final version of the manuscript.

\section{Acknowledgements}

We thank tea office of Guiding, Huishui, Liping, Renhuai, Sandu, Wuchuan, hishui, Daozhen, Dejiang,Duyun, Guian, Jinsha, Liuzhi, Nayong, Pu an, Puding ,Qinglong, Qixingguan, Sandu, Shiqian, Shuicheng, Tongzi, Wuchuan, Xingren, Xingyi, Xishui, Yanhe, Yinjiang, Yuqing, Zhenfeng, zheng'an for their help in teas collection. We thank College of tea science of Guizhou University and Department of Horticultural Sciences of Texas A\&M University for providing research facilities and computing facilities.

\section{References}

Arora, S., Cheema, J., Poland, J., Uauy, C., \& Chhuneja, P. (2019). Genome-wide association mapping of grain micronutrients concentration in aegilops tauschii. Frontiers in Plant Science, 10.

Babu, B.K., Sood, S., Chandrashekara, C., Pattanayak, A., and Kant, L. (2018). Mapping quantitative trait loci for important agronomic traits in finger millet (Eleusine coracana) mini core collection with genomic and genic SSR markers. Journal of Plant Biochemistry \& Biotechnology (1), 1-14.

Bac-Molenaar, J A., Fradin, E.F., Becker, F.M., Rienstra, J.A., Schoot, J., Vreugdenhil, D., Keurentjes J.J.B. (2015). Genome-wide association mapping of fertility reduction upon heat stress reveals Ddevelopmental stage-specific QTLs in Arabidopsis thaliana. The Plant Cell, 27(7): 1857-1874.

Brown, AHD. (1989). Core collections-a practical approach togenetic-resources management. Genome, 31(2):818-824.

Baker, R.L., Fung, L.W., Brock, M.T., Markelz, R.J.C., Covington, M.F., Devisetty, 
520

521

522

523

524

525

526

527

528

529

530

531

532

533

534

535

536

537

538

539

540

541

542

543

544

545

546

U.K.,Edwards, C.E., Maloof, J., Welch, S., Weinig, C. (2015). Modeling development and quantitative trait mapping reveal independent genetic modules for leaf size and shape. New Phytologist, 208 (1), 257-268.

Bali, S., Mamgain, A., Raina, S.N., Yadava, S.K., Bhat, V., Das, S., Pradhan, A.K., Goel, S. (2015). Construction of a genetic linkage map and mapping of drought tolerance trait in Indian beveragial tea. Molecular Breeding, 35 (5), 1-20.

Bartholomé, J., Bink, M.C., Van, H.J., Chancerel, E., Boury, C., Lesur, I., Isik, F., Bouffier, L., Plomion, C. (2016). Correction: Linkage and association mapping for two major traits used in the maritime pine breeding program: Height growth and stem straightness. Plos One ,11 (11).

Beukelaer, H. D., Smýkal, P., Davenport, G. F., and Fack, V. (2012). Core Hunter II: fast core subset selection based on multiple genetic diversity measures using Mixed Replica search. BMC Bioinformatics, 13:312.

Beukelaer, H. D., Davenport, G. F., Veetle, F. (2018). Core Hunter 3: flexible core subset selection. BMC Bioinformatics, 19:203. Bhattarai, U., and Subudhi, P.K. (2018). Identification of drought responsive QTLs during vegetative growth stage of rice using a saturated GBS-based SNP linkage map. Euphytica, 214 (2), 38.

Botstein, D., White, R, L., Skolnick, M., Davis, R.W. (2008). Construction of a genetic linkage map in man using restriction fragment length polymorphisms. American journal of human genetics, 32(3), 314-331.

Bouchet, S., Olatoye, M.O., Marla, S.R., Perumal, R., Tesso, T., Yu, J., Tuinstra, M., Morris, G.P. (2017). Increased power to dissect adaptive traits in global sorghum diversity using a nested association mapping population. Genetics ,206 (2), 573-585.

Bradbury, P.J., Zhang, Z., Kroon, D.E., Casstevens, T.M., Ramdoss, Y., and Buckler, E.S. (2007). TASSEL: software for association mapping of complex traits in diverse samples. Bioinformatics, 23 (19), 2633-2635.

Brown, A. H. D. (1989). Core collections: a practical approach to genetic resources management. 
547

548

549

550

551

552

553

554

555

556

557

558

559

560

561

562

563

564

565

566

567

568

569

570

571

572

573

Genome, 31, 818-824. Brown, A.H.D., Hintum, V., Th., J.L., Morales, and E., A.V. (2000). Core collections of plant genetic resources. IPGRI Technical Bulletin No.3. International Plant Genetic Resources Institute, Rome, Italy, 48 pp (96).

Buckler, E.S., and Thornsberry, J.M. (2002). Plant molecular diversity and applications to genomics. Current Opinion in Plant Biology 5 (2), 107-111.

Campoy, J.A., Lerigoleurbalsemin, E., Christmann, H., Beauvieux, R., Girollet, N., Querogarcía, J., Dirlewanger, E., Barreneche, T. (2016). Genetic diversity, linkage disequilibrium, population structure and construction of a core collection of Prunus avium L. landraces and bred cultivars. BMC Plant Biology , 16 (1), 49.

Chen, L., Yu, F.L., and Tong, Q.Q. (2000). Discussions on phylogenetic classification and evolution of Sect.Thea. Journal of Tea Science, 20 (2): 89-94.

Chen, L., Apostolides, Z., and Chen, Z.M. (2012). Global tea breeding: Achievements, challenges and perspectives. Springer-Zhejiang University Press

Chen, W., Hou, L., Zhang, Z., Pang, X., and Li, Y. (2017). Genetic diversity, population structure, and linkage disequilibrium of a core collection of Ziziphus jujuba assessed with genome-wide SNPs developed by genotyping-by-sequencing and SSR markers. Front Plant Sci $, 8,575$.

Claire, B., Punna, R., Sophie, B., Jacques, C., Monique, D., Laetitia, G., Jean-Louis, N., JeanFran?Ois, R., Ronan, R., Yu, L. (2013). Massive sorghum collection genotyped with SSR markers to enhance use of global genetic resources. Plos One, 8 (4).

Coan, M.M.D., Senhorinho, H.J.C., Pinto, R.J.B., Scapim, C.A., and Warburton, M.L. (2018). Genome-wide association study of resistance to ear rot by in a tropical field maize and popcorn core collection. Crop Science, 58 (2), 564-.

Cunff, L.L., Fournier-Level, A., Laucou, V., Vezzulli, S., Lacombe, T., Adam-Blondon, A.F., Boursiquot, J.M., This, P. (2008). Construction of nested genetic core collections to optimize the exploitation of natural diversity in Vitis vinifera L. subsp. sativa. BMC Plant Biology, 8 (1), 31. 
574 Desgroux, A., Baudais, V.N., Aubert, V., Le, R.G., De, L.H., Miteul, H., Aubert, G., Boutet, G., 575 Duc, G., Baranger, A. (2017). Comparative genome-wide-association mapping identifies 576 common loci controlling root system architecture and resistance to aphanomyces euteiches in pea. Frontiers in Plant Science, 8, 2195.

Egbadzor, K.F., Ofori, K., Yeboah, M., Aboagye, L.M., Opoku-Agyeman, M.O., Danquah, E.Y., Offei, S.K. (2014). Diversity in 113 cowpea [ Vigna unguiculata (L) Walp] accessions assessed with 458 SNP markers. Springer plus ,3 (1), 541.

Elshire, R.J., Glaubitz, J.C., Sun, Q., Poland, J.A., Kawamoto, K., Buckler, E.S., Mitchell, S.E. (2011) A robust, simple genotyping-by-sequencing (GBS) approach for high diversity species. PloS One ,6 (5).

584

585

586

587

588

589

590

591

592

593

594

595

596

597

598

599

600

Eltaher, S., Sallam, A., Belamkar, V., Emara, H., Nower, A., Salem, K., Poland, J., and Baenziger, P.S. (2018). Genetic diversity and population structure of F Nebraska winter wheat genotypes using genotyping-by-sequencing. Front Genet, 9, 76.

Franco, J., Crossa, J., Taba, S., and Shands, H. (2005). A sampling strategy for conserving genetic diversity when forming core subsets. Crop Science ,45 (3), 1035-1044.

Frankel, OH. (1984). Genetic perspectives of germplasm conservation. In: Arber, W.K., Llimensee, K., Peacock, W.J., Starlinger, P. (eds) Genetic manipulation: impact on manand society. Cambridge University Press, Cambridge, England, 161-170.

Gao, X. Y., Joshua, S., and Martin, E.R. (2010). A multiple testing correction method for genetic association studies using correlated single nucleotide polymorphisms. Genetic Epidemiology ,32 (4), 361-369.

Hackett, C.A., Milne, L., Smith, K., Hedley, P., Morris, J., Simpson, C.G., Preedy, K., Graham, J. (2018). Enhancement of Glen Moy x Latham raspberry linkage map using GBS to further understand control of developmental processes leading to fruit ripening. BMC Genetics, 19 .

Hamon, P., Seguin, M., Perrier, X., and Glaszmann, J.C. (2003). Genetic diversity of cultivated tropical plants. Diversite Genetique Des Plantes Tropicales Cultivees.

Hayat, K., Iqbal, H., Malik, U., Bilal, U., and Mushtaq, S. (2015). Tea and its consumption: 
601

602

603

604

605

606

607

608

609

610

611

612

613

614

615

616

617

618

619

620

621

622

623

624

625

626

627

benefits and risks. Crit Rev Food Sci Nutr, 55 (7), 939-954.

Heena, A., Shivendra, K., Amar, K., Manu, A., Arun, J., \& Shailendra, G. (2018). Association mapping for important agronomic traits in safflower (carthamus tinctorius L.) core collection using microsatellite markers. Frontiers in Plant Science, 9, 402-.

Hou, J., Zhou, Y.-F., Gao, L.-Y., Wang, Y.-L., Yang, L.-M., Zhu, H.-Y., Wang, J.-M., Z Zhao, S.-J., $\quad$ Ma, C.-S., $\quad$ Sun, S.-R., Hu, J.-B. (2018). Dissecting the genetic architecture of melon chilling tolerance at the seedling stage by association mapping and identification of the elite alleles. Frontiers in Plant Science ,9 (1577).

Huggins, T.D., Chen, M.-H., Fjellstrom, R.G., Jackson, A.K., McClung, A.M., and Edwards, J.D. (2018). Association analysis of three diverse rice (Oryza sativa L.) germplasm collections for loci regulating grain quality traits. The Plant Genome. doi:10.3835/plantgenome2017.09.0085

Hussain, W., Baenziger, P.S., Belamkar, V., Guttieri, M.J., Venegas, J.P., Easterly, A., Sallam, A., Poland, J. (2017). Genotyping-by-sequencing derived high-density linkage map and its application to QTL mapping of flag leaf traits in bread wheat. Sci Rep, 7 (1).

Iso-Touru, T., Sahana, G., Guldbrandtsen, B., Lund, M.S., and Vilkki, J. (2016). Genome-wide association analysis of milk yield traits in Nordic Red Cattle using imputed whole genome sequence variants. BMC Genetics, 17 (1), 55.

Jin J, Chai Y, Liu Y, Zhang J, Yao M, Chen L. (2018). Hongyacha, a naturally caffeine-free tea plant from Fujian, China. Journal of agricultural and food chemistry, 100:10.

Lassois, L., Denancé, C., Ravon, E., Guyader, A., Guisnel, R., Hibrandsaintoyant, L., Poncet, C., Lasserrezuber, P., Feugey, L., Durel, C.E. (2016). Genetic diversity, population structure, parentage analysis, and construction of core collections in the French apple germplasm based on SSR markers. Plant Molecular Biology Reporter ,34 (4), 827-844.

Li, D., Zhao, X., Han, Y., Li, W., and Xie, F. (2018). Genome-wide association mapping for seed protein and oil contents using a large panel of soybean accessions. Genomics. doi: 10.1016/j.ygeno.2018.01.004 
628

629

630

631

632

633

634

635

636

637

638

639

640

641

642

643

644

645

646

647

648

649

650

651

652

653

654

Li, H. (2013). Aligning sequence reads, clone sequences and assembly contigs with BWA-MEM. arXiv:1303.3997.

Liang, Y.R., and Shi, M. (2015). Advances in tea plant genetics and breeding. Journal Tea Science, 35:103-109.

Liu, S., Liu, H., Wu, A., Hou, Y., An, Y., and Wei, C. (2017). Construction of fingerprinting for tea plant (Camellia sinensis) accessions using new genomic SSR markers. Molecular Breeding, 37 (8).

Thierry, L., Fabien, D.B., Hyacinthe, L., Pascal, M., Adrien, K., Rey Gastón, L.S., Philippe, C. (2014). Developing core collections to optimize the management and the exploitation of diversity of the coffee Coffea canephora. Genetica, 142, 185-199.

Luo, X., Ma, C., Yue, Y., Hu, k., Li, Y., Duan, Z., Wu, M.,Tu, J., Shen, J., Yi, B., Fu, T.(2015). Unravelling the complex trait of harvest index in rapeseed (Brassica napus L.) with association mapping. BMC Genomics, 16:379.

Ma, Z., He, S., Wang, X., Sun, J., Zhang, Y., Zhang, G., Wu, L., Li, Z., Liu, Z., and Sun, G. (2018). Resequencing a core collection of upland cotton identifies genomic variation and loci influencing fiber quality and yield. Nature Genetics, 50 (6).

Ma, J.Q., Jin, J.J., Yao, M.Z., Ma,C.L., Xu,Y.X., Hao, W.G., Chen, L. (2018). Quantitative trait loci mapping for theobromine and caffeine contents in tea plant (Camellia sinensis). Journal of Agricultural and Food Chemistry, 66 (50), 13321-13327.

Ma, Q., Chen, C., Zeng, Z., Zou, Z., Li, H., Zhou, Q., Chen, X., Sun, K., Li, X. (2018). Transcriptomic analysis between self- and cross-pollinated pistils of tea plants (Camellia sinensis). BMC Genomics, 19:289.

Mckenna, A., Hanna, M.E., Sivachenko, A., Cibulskis, K., Kernytsky, A., Garimella, K., Altshuler, D., Gabriel, S., Daly, M., Depristo, M.A. (2010). The genome analysis toolkit: a map reduce framework for analyzing next-generation DNA sequencing data. Genome Res, 20, 1297-1303.

Min, T. L. (1992). A revision of Camellia sect.thea. Acta Botanica Yunnanica ,14 (2): 115-132. 
655

656

657

658

659

660

661

662

663

664

665

666

667

668

669

670

671

672

673

674

675

676

677

678

679

680

681

Motilal, L.A., Zhang, D., Mischke, S., Meinhardt, L.W., Boccara, M., Fouet, O., Lanaud, C., Umaharan, P. (2016). Association mapping of seed and disease resistance traits in Theobroma cacao L. Planta, 244 (6), 1265-1276.

Muleta, K.T., Bulli, P., Zhang, Z., Chen, X., and Pumphrey, M. (2017). Unlocking diversity in germplasm collections via genomic selection: a case study based on quantitative adult plant resistance to stripe rust in spring wheat. Plant Genome, 10 (3).

Naghma, K., and Hasan, M. (2013). Tea and health: studies in humans. Current Pharmaceutical Design, 19 (34).

Ndjiondjop, M., Semagn, K., Gouda, A., Kpeki, S., Dro Tia, D., Sow, M., Goungoulou, A., Sie, M., Perrier, X., Ghesquiere, A. (2017). Genetic variation and population structure of and development of a mini-core collection using DArTseq. Front Plant Science, 8, 1748.

Niu, S.Z. (2014). Studies on genetic diversity and resistance of wild tea germplasm (Camellia spp.) in Guizhou province. [doctoral thesis]: Guizhou university.

Niu, S., Song, Q,Koiwa,H., Qiao, D., Zhao, D., Chen, Z., Liu, X., Wen,X. (2019) . Genetic diversity, linkage disequilibrium, and population structure analysis of the tea plant (Camellia sinensis) from an origin center, Guizhou plateau, using genome wide SNPs developed by genotyping-by sequencing. BMC Plant Biology , 19:328.

Odong, T.L., Jansen, J., Eeuwijk, F.A., Van, and Hintum, T.J.L., Van (2013). Quality of core collections for effective utilisation of genetic resources review, discussion and interpretation. Theoretical \& Applied Genetics, 126 (2), 289-305.

Pace, J., Gardner, C., Romay, C., Ganapathysubramanian, B., and Lübberstedt, T. (2015). Genome-wide association analysis of seedling root development in maize (Zea mays L.). BMC Genomics, 16 (1) , 47.

Song, J. M., Arif, M., Zhang, M., Sze, S.H., Zhang, H.B. (2019). Phenotypic and molecular dissection of grain quality using the USDA rice mini-core collection. Food Chemistry, 284 (30):312-322.

Suwarno, W.B., Pixley, K.V., Palacios-Rojas, N., Kaeppler, S.M., and Babu, R. (2015). 
682

683

684

685

686

687

688

689

690

691

692

693

694

695

696

697

698

699

700

701

702

703

704

705

706

707

708

Genome-wide association analysis reveals new targets for carotenoid biofortification in maize. Theoretical \& Applied Genetics, 128 (5), 851-864.

Taniguchi, F., Kimura, K., Saba, T. (2014) Worldwide core collections of tea (Camellia sinensis) based on SSR markers. Tree Genetics \& Genomes, 10, 1555-1565.

Tan, L.Q., Wang, L.Y., Xu, L.Y., Wu, L.Y., Peng, M., Zhang, C.C., Wei, K., Bai, P.-X., Li, H.L., Cheng, H., Qi, G. N. (2016). SSR-based genetic mapping and QTL analysis for timing of spring bud flush, young shoot color, and mature leaf size in tea plant (Camellia sinensis). Tree Genetics \& Genomes, 12 (3), 52.

Taniguchi, I., McCloskey, A., Ohno, M. (2014). Chapter 18 - Analysis of RNA transport in xenopus oocytes and mammalian cells. Methods in Cell Biology, 122: 395-413.

Wambulwa, M.C., Meegahakumbura, M.K., Kamunya, S., Muchugi, A., Moller, M., Liu, J., Xu, J.C., Ranjitkar, S., Li, D.Z., Gao, L.M. (2016). Insights into the genetic relationships and breeding patterns of the African tea germplasm based on nSSR markers and cpDNA sequences. Front Plant Science, 7, 1244.

Wang, X.C., Liu, Z., Yao, M.Z., Ma, C.L., Chen, L., Yang, Y.J. (2009). Sampling strategy to establish a primary core collection of Chinese tea germplasms. Journal of Tea Science, 29 (2),159-167.

Wang, XC., Chen, L., Yang, YJ (2011). Establishment of core collection for Chinese tea germplasm based on cultivated region grouping and phenotypic data. Front. Agric. China, 5(3): 344-350.

Wang, Y., Song, F., Zhu, J., Zhang, S., Yang, Y., Chen, T., Tang, B., Dong, L., Ding, N., Zhang, Q., $\quad$ Bai, Z., $\quad$ Dong, X., $\quad$ Chen, H., Sun, M., Zhai, S., Sun, Y., Yu, L., Lan, L., Xiao, J., Fang, X., Lei, H., Zhang, Z., Zhao, W. (2017). GSA: Genome sequence archive*. Genomics, Proteomics \& Bioinformatics, 15 (1), 14-18.

Wei, C., Yang, H., Wang, S., Zhao, J., Liu, C., Gao, L., Xia, E., Lu, Y., Tai, Y., She, G., Sun, J., Cao, H., Tong, W., Gao, Q., Li, Y., Deng, W., Jiang, X., Wang, W., Chen, Q., Zhang, S., Li, H., Wu, J., Wang, P., Li, P., Shi, C., Zheng, F., Jian, 
709

710

711

712

713

714

715

716

717

718

719

720

721

722

723

724

725

726

727

728

729

730

731

732

733

J., Huang, B., Shan, D., Shi, M., Fang, C., Yue, Y., Li, F., Li, D., Wei, S., Han, B., Jiang, C., Yin, Y., Xia, T., Zhang, Z., Bennetzen, J.L., Zhao, S., Wan, X. (2018). Draft genome sequence of Camellia sinensis var. sinensis provides insights into the evolution of the tea genome and tea quality. Proc Natl Acad Sci U S A, 115 (18), E4151E4158.

Xia, E.H., Zhang, H.B., Sheng, J., Li, K., Zhang, Q.J., Kim, C., Zhang, Y., Liu, Y., Zhu, T., Li, W., Huang, H., Tong, Y., Nan, H., Shi, C., Shi, C., Jiang, J.J., Mao, S.Y., Jiao, J.Y., Zhang, D., Zhao, Y., Zhao, Y.J., Zhang, L.P., Liu, Y.L., Liu, B.Y., Yu, Y., Shao, S.F., Ni, D.J., Eichler, E.E., Gao, L.Z. (2017). The tea tree genome provides insights into tea flavor and independent evolution of caffeine biosynthesis. Mol Plant, 10 (6), 866-877.

Yang, Z., Chen, Z., Peng, Z., Yu, Y., Liao, M., and Wei, S. (2017). Development of a highdensity linkage map and mapping of the three-pistil gene (Pis1) in wheat using GBS markers. BMC Genomics, 18 (1), 567.

Yao, M.-Z., Ma, C.-L., Qiao, T.-T., Jin, J.-Q., and Chen, L. (2012). Diversity distribution and population structure of tea germplasms in China revealed by EST-SSR markers. Tree Genetics \& Genomes, 8 (1), 205-220.

Zhang, D.Z., Rabia, B.P., Liu, J.J., Gong, X.W., Liang, J.B., Liu, M.X., Lu, P., Gao, X. L., Feng, B.L. (2018). Morphological diversity and correlation analysis of phenotypes and quality traits of proso millet (Panicum miliaceum L.) core collections. Journal of Integrative Agriculture, 17 (0), 60345-60347.

Zhao, X., Dong, H., Chang,H., Zhao,J., Teng,W., Qiu, L., Li, W., Han,Y (2019). Genome wide association mapping and candidate gene analysis for hundred seed weight in soybean [Glycine $\max ($ L.) Merrill]. BMC Genomics, 20:648. 


\section{Figure 1}

Frequency distribution categories of pairwise genetic distance of 415 tea accessions

Frequency distribution categories of pairwise genetic distance of 415 tea accessions (red fill); a core set of 299 accessions (orange fill); a core set of 198 accessions (green fill), and a minicore set of 148 accessions (purple fill), based on 30,282 polymorphic SNPs.

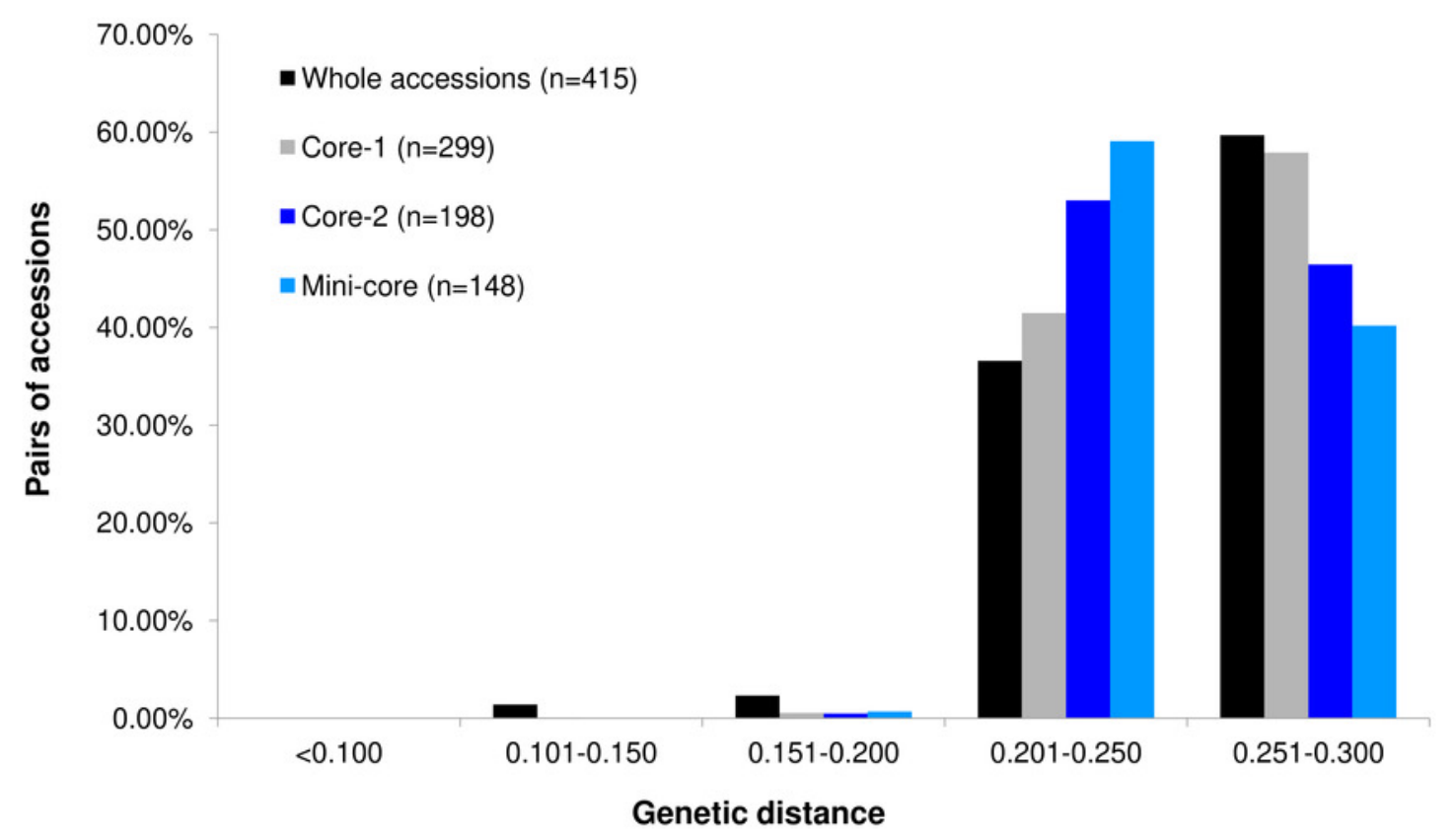




\section{Figure 2}

Neighbor joining tree

Neighbor joining tree for (A) 415 accessions (G1 = Group 1, pink; G2 = Group 2, red;G3= Group 3, light green; G4 = Group 4, blue; G5 = Group 5, brown ; G6 = Group 6 , orange red; G7 = Group 7 , orange yellow; G8 = Group 8, light blue; G9 = Group 9 ,yellow; G10 = Group 10, light purple; G11= Group 11, dark green; G12 = Group12, dark blue; G13 = Group 13, black; G14 = Group 14, purple), (B) 299 accessions selected for a core set, (C) 198 accessions selected for a core set and (D) 148 accessions selected for a mini-core set based on 30282 polymorphic SNPs. 

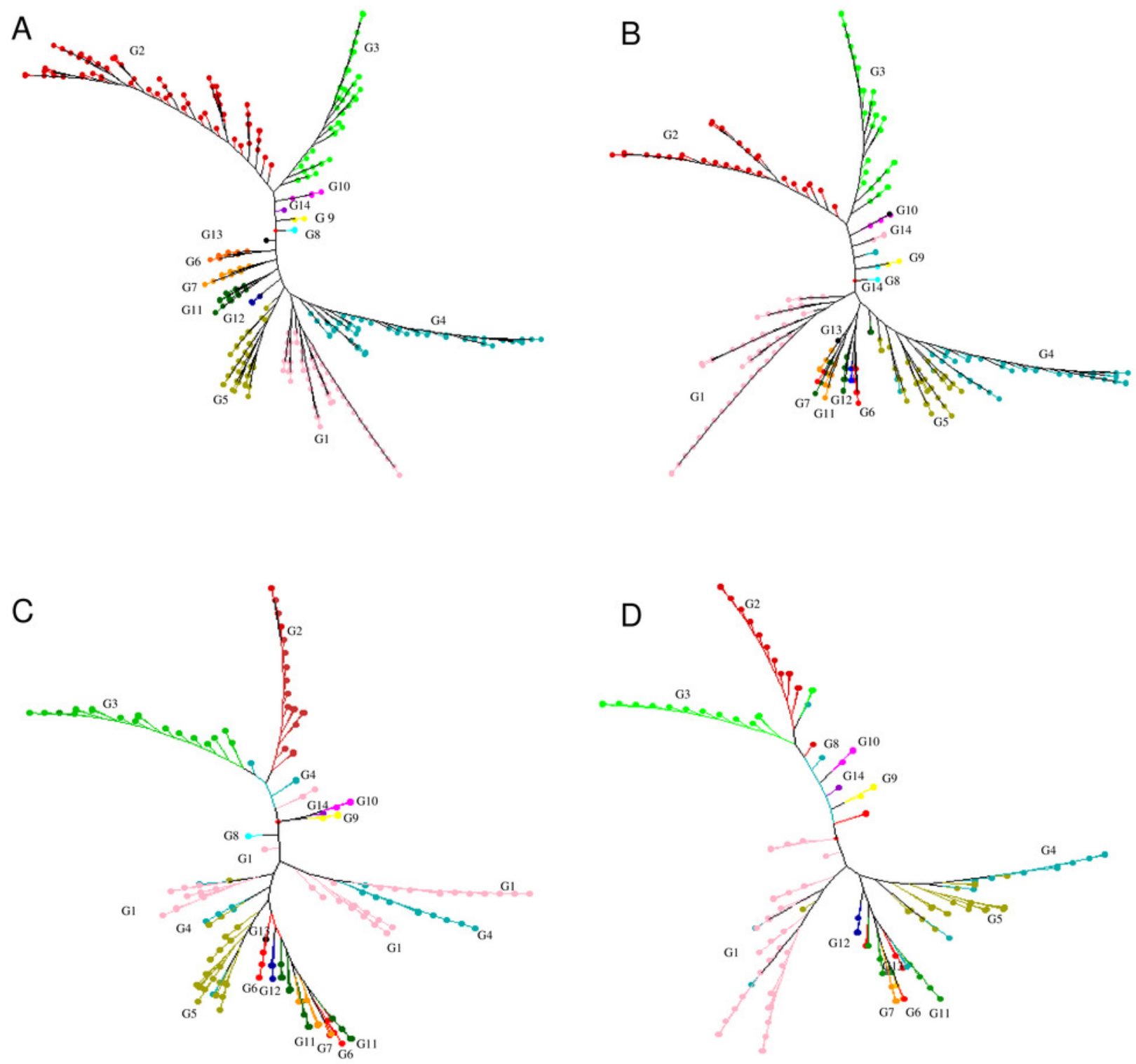


\section{Figure 3}

Summary of accessions elected to conform core-1, core-2 and mini-core

Summary of accessions elected to conform core-1, core-2 and mini-core collection compared with the whole tea accessions by (A) groups predicted based on cluster analysis ( $G 1=$ Group 1,G2 = Group 2, G3= Group 3, G4 = Group 4, G5 = Group 5, G6 = Group 6 , G7 = Group 7 , $\mathrm{G} 8=$ Group 8, G9 = Group 9 , G10 = Group 10, G11= Group 11, G12 = Group 12, G13 = Group 13, G14 = Group 14) ; (B) summary of accessions selected to account for core-1, core-2 and mini-core collection compared with the whole tea accessions in each group by cultivation status (AL, Ancient landraces; ML, Modern landraces; WT, Wild tree ), by Growth Habits (CT, cultivation type; WT, wild type) and Species of origin (S1, C.sinensis; S2, C.tachangensis; S3, C.remotiserrata; S4, C.taliensis). 

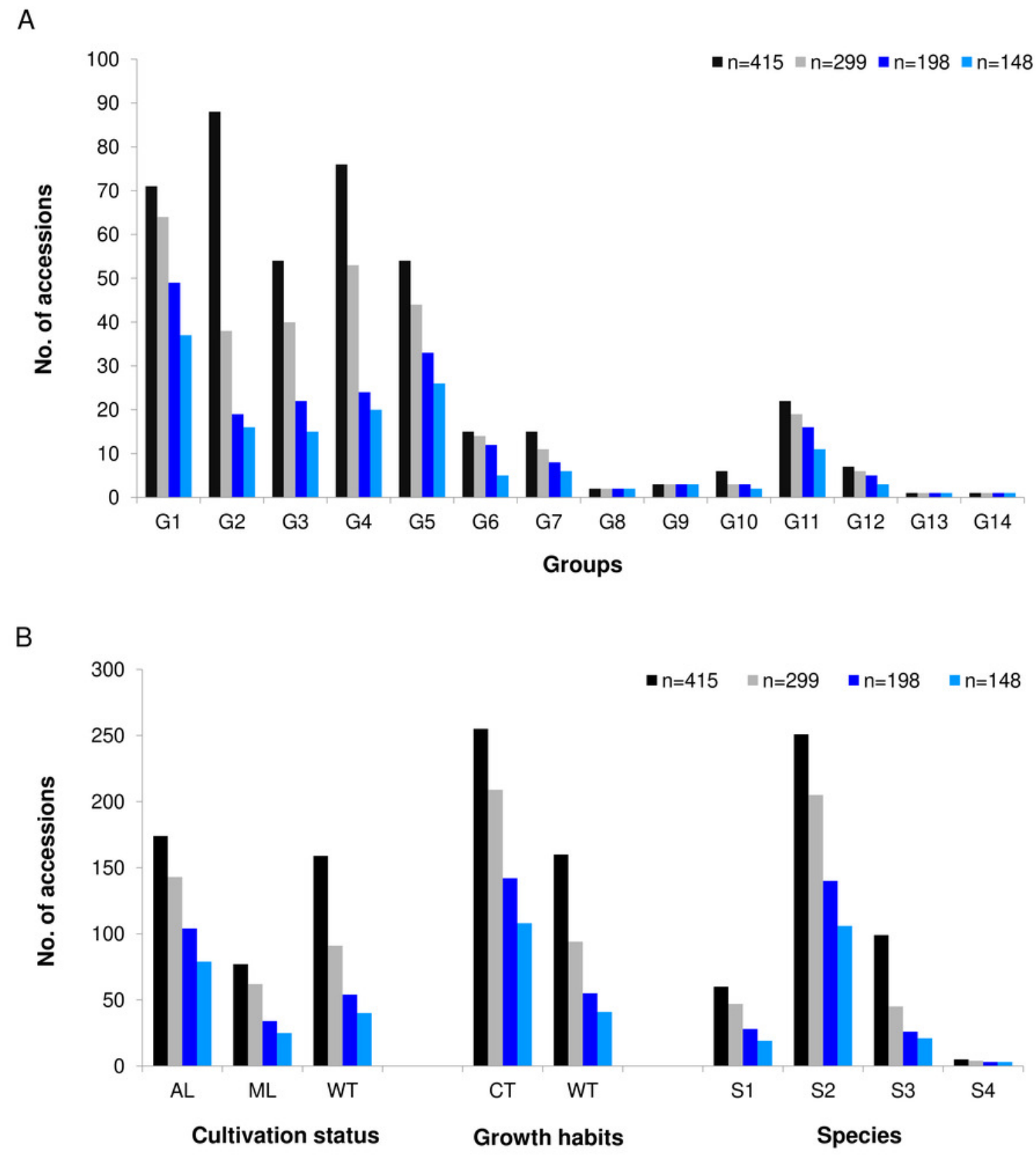


\section{Table $\mathbf{1}$ (on next page)}

Genetic differentiation of inferred populations of tea accessions from Guizhou Province.

Genetic differentiation of inferred populations of tea accessions from Guizhou Province. 
1 Table 1 Genetic differentiation of core and mini-core sets of tea plant in Guizhou Province.

\begin{tabular}{llllllll}
\hline Group & S & ASC & Ho & PIC & MAF & GDR & AGD \\
\hline Whole Set & 415 & 26,810 & $0.215 \pm 0.006 \mathrm{a}$ & $0.359 \pm 0.006 \mathrm{a}$ & $0.171 \pm 0.002 \mathrm{a}$ & $0.088-0.298$ & 0.249 \\
Core-1 & 299 & 26,592 & $0.223 \pm 0.005 \mathrm{a}$ & $0.360 \pm 0.008 \mathrm{a}$ & $0.172 \pm 0.001 \mathrm{a}$ & $0.110-0.298$ & 0.252 \\
Core-2 & 198 & 24,917 & $0.206 \pm 0.006 \mathrm{a}$ & $0.363 \pm 0.006 \mathrm{a}$ & $0.170 \pm 0.001 \mathrm{ab}$ & $0.115-0.298$ & 0.250 \\
Mini-Core & 148 & 23,731 & $0.193 \pm 0.006 \mathrm{~b}$ & $0.355 \pm 0.005 \mathrm{a}$ & $0.168 \pm 0.001 \mathrm{~b}$ & $0.115-0.292$ & 0.248 \\
\hline
\end{tabular}

2 Note: S, Sample size; ASC, Average Site Count; Ho, observed heterozygosity; PIC, polymorphism

3 information content; MAF, Minor Allele Frequency; GDR, Genetic distance range; AGD, average genetic 4 distance. The different letters indicate a significant difference in a column at $p=0.05$ levels by T-test. 


\section{Table 2 (on next page)}

The number of whole accessions and core collections distributed in 14 groups.

The number of whole accessions and core collections distributed in 14 groups. 
1 Table 2 The number of whole accessions and core collections distributed in 14 groups

\begin{tabular}{lllll}
\hline Group & $\begin{array}{l}\text { Number of } \\
\text { accessions of } \\
\text { whole set }\end{array}$ & $\begin{array}{l}\text { Number of accessions } \\
\text { retained in the 299 core } \\
\text { set }\end{array}$ & $\begin{array}{l}\text { Number of accessions } \\
\text { retained in the 198 } \\
\text { core set }\end{array}$ & $\begin{array}{l}\text { Number of accessions } \\
\text { retained in the 148 } \\
\text { mini-core set }\end{array}$ \\
\hline Group1 & 71 & 64 & 49 & 37 \\
Group2 & 88 & 38 & 19 & 16 \\
Group3 & 55 & 40 & 22 & 15 \\
Group4 & 75 & 53 & 24 & 20 \\
Group5 & 54 & 44 & 33 & 26 \\
Group6 & 15 & 14 & 12 & 5 \\
Group7 & 15 & 11 & 8 & 6 \\
Group8 & 2 & 2 & 2 & 2 \\
Group9 & 3 & 3 & 3 & 3 \\
Group10 & 6 & 3 & 3 & 2 \\
Group11 & 22 & 19 & 16 & 11 \\
Group12 & 7 & 6 & 5 & 3 \\
Group13 & 1 & 1 & 1 & 1 \\
Group14 & 1 & 1 & 1 & 1 \\
Total & 415 & 299 & 198 & 148 \\
\hline
\end{tabular}




\section{Table 3 (on next page)}

Trait statistics collected for mature leaf length (MLL), mature leaf width (MLW), mature leaf shape index (MLSI) and mature leaf area (MLA).

Trait statistics collected for mature leaf length (MLL), mature leaf width (MLW), mature leaf shape index (MLSI) and mature leaf area (MLA). 
1 Table 3 Trait statistics collected for mature leaf length (MLL), mature leaf width (MLW), mature leaf shape

2 index (MLSI) and mature leaf area (MLA)

\begin{tabular}{lllllcc}
\hline Trait & Mean & Std. dev & Minimum & Maximum & Skewness (Std. Err) & Kurtosis ( Std. Err) \\
\hline MLL & 8.655 & 2.251 & 3.886 & 15.200 & $0.497(0.122)$ & $0.002(0.243)$ \\
MLW & 3.748 & 0.933 & 1.986 & 6.830 & $0.687(0.122)$ & $0.415(0.244)$ \\
MLA & 25.700 & 12.929 & 6.570 & 75.566 & $1.223(0.122)$ & $1.582(0.244)$ \\
MLI & 2.326 & 0.316 & 0.830 & 3.457 & $0.043(0.122)$ & $1.949(0.244)$ \\
\hline
\end{tabular}

3

4

5

6

7

8

9

10

11

12

13

14

15 


\section{Table 4(on next page)}

The correlation coefficient between two environment and among four traits.

The correlation coefficient between two environment and among four traits. 
1

2 Table 4 The correlation coefficient between two environment and among four traits

\begin{tabular}{lllll}
\hline Trait/ Environment & MLL & MLW & MLA & M1SI \\
\hline MLL & $0.896 * *$ & & & \\
MLW & $0.857 * *$ & $0.960 * *$ & & \\
MLA & $0.953 * *$ & $0.951 * *$ & $0.905 * *$ & \\
MLSI & $0.338 * *$ & $-0.174 * *$ & 0.081 & $0.806 * *$ \\
\hline
\end{tabular}

3 Note: The diagonal line is the correlation coefficient of two environments; * indicate a significant difference in a column at $p=$

$4 \quad 0.05$ levels, ** indicate a significant difference in a column at $p=0.01$ levels

5

6

7

8

9

10

11

12 


\section{Table 5 (on next page)}

SNPs significantly associated with mature leaf length (MLL), mature leaf width (MLW), mature leaf shape index (MLSI) and mature leaf area (MLA) detected by GWAS of 415 accessions

SNPs significantly associated with mature leaf length (MLL), mature leaf width (MLW), mature leaf shape index (MLSI) and mature leaf area (MLA) detected by GWAS of 415 accessions 
1 Table 5 SNPs significantly associated with mature leaf length (MLL), mature leaf width (MLW), mature leaf shape index (MLSI) and mature leaf area 2 (MLA) detected by GWAS of 415 accessions

3 Note: Pre-Group, Pseudo Group; MAF minor allele frequency.

\begin{tabular}{|c|c|c|c|c|c|c|c|c|c|c|c|}
\hline Trait & SNP Marker & Pse-Group & Position(bp) & Scaffold & $\begin{array}{l}\text { Major / } \\
\text { minor } \\
\text { allele }\end{array}$ & MAF & $P$-value & $\begin{array}{l}\text { Major/minor } \\
\text { allele effect }\end{array}$ & $R$-square & $\begin{array}{l}\text { No. of accessions } \\
\text { with homozygous } \\
\text { major/minor allele }\end{array}$ & $\begin{array}{l}\text { No. of accessions } \\
\text { with heterozygous } \\
\text { allele }\end{array}$ \\
\hline \multirow[t]{2}{*}{ MLL } & PG_13:79474508 & 13 & 79474508 & xfSc0000122 & $\mathrm{G} / \mathrm{T}$ & 0.07 & $5.41 \mathrm{E}-07$ & $-0.72 /-3.36$ & $3.77 \%$ & $307 / 14$ & 22 \\
\hline & PG_1:32346865 & 1 & 32346865 & Sc0000000 & $\mathrm{T} / \mathrm{A}$ & 0.05 & $1.03 \mathrm{E}-06$ & $-2.94 / 1.09$ & $3.23 \%$ & $345 / 8$ & 23 \\
\hline MLW & PG_1:32346865 & 1 & 32346865 & $\operatorname{Sc} 0000000$ & $\mathrm{~T} / \mathrm{A}$ & 0.05 & $3.59 \mathrm{E}-07$ & $-1.31 / 0.44$ & $8.24 \%$ & $344 / 8$ & 23 \\
\hline \multirow[t]{6}{*}{ MLA } & PG_1:32346865 & 1 & 32346865 & $\operatorname{Sc} 0000000$ & $\mathrm{~T} / \mathrm{A}$ & 0.05 & $1.95 \mathrm{E}-08$ & $-18.23 / 8.25$ & $4.50 \%$ & $344 / 8$ & 23 \\
\hline & PG_4:40545730 & 4 & 40545730 & Sc0000365 & $\mathrm{G} / \mathrm{A}$ & 0.07 & $8.80 \mathrm{E}-07$ & $-1.47 / 14.72$ & $3.79 \%$ & $316 / 13$ & 20 \\
\hline & PG_12:21050315 & 12 & 21050315 & Sc0002172 & $\mathrm{C} / \mathrm{T}$ & 0.06 & $9.47 \mathrm{E}-07$ & $-4.12 / 11.61$ & $3.58 \%$ & $327 / 19$ & 8 \\
\hline & PG_12:133979444 & 12 & 133979444 & $\operatorname{Sc} 0002452$ & $\mathrm{G} / \mathrm{A}$ & 0.06 & $1.07 \mathrm{E}-06$ & $1.06 / 42.47$ & $3.49 \%$ & $322 / 2$ & 36 \\
\hline & PG_16:74477057 & 16 & 74477057 & Sc0004093 & $\mathrm{G} / \mathrm{A}$ & 0.08 & $1.40 \mathrm{E}-06$ & $4.46 / 31.33$ & $3.81 \%$ & $283 / 4$ & 42 \\
\hline & PG_13:79474508 & 13 & 79474508 & $\mathrm{xfSc} 0000122$ & $\mathrm{G} / \mathrm{T}$ & 0.07 & $1.45 \mathrm{E}-06$ & $-5.57 /-18.76$ & $3.68 \%$ & $306 / 14$ & 22 \\
\hline \multirow[t]{3}{*}{ MLI } & PG_13:82813415 & 1 & 82813415 & $\operatorname{Sc} 0000037$ & $\mathrm{~T} / \mathrm{C}$ & 0.13 & $3.24 \mathrm{E}-07$ & $-0.08 /-1.25$ & $4.01 \%$ & $266 / 1$ & 96 \\
\hline & PG_17:30871283 & 17 & 30871283 & Sc0000011 & $A / G$ & 0.05 & $5.84 \mathrm{E}-07$ & $-1.49 / 0.09$ & $4.33 \%$ & $280 / 1$ & 32 \\
\hline & PG_10:98891957 & 10 & 98891957 & Sc0000046 & $\mathrm{G} / \mathrm{A}$ & 0.06 & $1.40 \mathrm{E}-06$ & $0.06 /-1.50$ & $3.83 \%$ & $306 / 1$ & 36 \\
\hline
\end{tabular}

4
5
6
7
8
9
10

